\title{
REVISIÓN
}

\section{Historia e investigación de la leucemia en Costa Rica}

\author{
Rafael Jiménez Bonilla ${ }^{1,2}$ \\ 1 Laboratorio de Investigación, Hospital Nacional de Niños. \\ 2 Facultad de Microbiología, Universidad de Costa Rica. 2060 San José, Costa Rica.
}

Recibido 05-XI-2003. Corregido 19-II-2004. Aceptado 09-III-2004.

\begin{abstract}
History of leukemia research in Costa Rica. A review of leukemia worldwide is discussed, focusing on etiology, diagnosis and treatment. The history of research of this type of cancer in Costa Rica is presented through the first hospital diagnosis, the arrival of clinical and laboratory hematologists, the establishment of specialized laboratories, the local hematology teaching programs and the voluntary associations that help patients with leukemia. A brief review of Costa Rican publications in this area and the future of this problem in our country is also shown. Rev. Biol. Trop. 52(3): 559-569. Epub 2004 Dic 15.
\end{abstract}

Key words: leukemia, leukemia history, hematology, genetics, Costa Rica.

Palabras clave: leucemia, investigación, historia, hematología, genética, Costa Rica.

La leucemia fue descubierta en el mundo hace casi 200 años, por lo que puede considerarse un problema reciente comparado con otros grandes flagelos de la humanidad. Desde su descripción inicial, las leucemias han sido foco de una impresionante investigación médica, debido a varias razones: sus dramáticas manifestaciones clínicas, su frecuencia e impacto en la niñez, su hallazgo en animales, la sencillez de los análisis de la sangre periférica y de la médula ósea que permitieron comprender mejor la hematopoyesis, y su forma de tratamiento, adaptada posteriormente a otros tipos de neoplasias en el ser humano. Sin embargo, el aspecto cumbre de su estudio fue el descubrimiento, hace 30 años, de que la variedad más común en el niño, la leucemia linfocítica aguda, podía curarse en al menos la mitad de los casos (Aur et al. 1971), la primera vez que esto se conseguía en una neoplasia generalizada.

\section{DESCRIPCIÓN MUNDIAL}

La leucemia fue descubierta casi al mismo tiempo por dos médicos brillantes que llevaron a cabo estudios cuidadosos, tanto en sus enfermos como en autopsias (Dameshek y Gunz 1964). El primero en describir esta enfermedad fue Velpeau en 1827, el cual observó un paciente de 63 años con fiebre, debilidad y un crecimiento descomunal del abdomen. Él encontró en la autopsia un hígado y un bazo enormes (el bazo pesó 4 kilos), y la "sangre era como una papilla de avena que recordaba la consistencia y el color de las levaduras del vino tinto" (Velpeau 1827).

Por otra parte, Barth (1856) estudió en 1839 un paciente cuya sangre fue analizada por Donné (1844), el cual observó en la autopsia al microscopio, unos "glóbulos mucosos muy parecidos a las células del pus". De acuerdo con esta secuencia, Donné habría sido la primera persona que describió microscópicamente las células leucémicas.

Los estudios iniciales de pacientes vivos con leucemia se hicieron en 1845 por tres investigadores de la época: Virchow (1845) en Alemania, el cual llamó a la enfermedad sangre blanca y fue el primero en proponer que el problema no era infeccioso sino una patología diferente que afectaba a ciertos órganos, y Bennett (1845) y Craigie (1845) en Escocia, 
los cuales reconocieron también la entidad como un problema propio de los glóbulos blancos. Virchow (1856), quien llegaría a ser uno de los grandes hombres en la patología mundial, introdujo dos años después el término leucemia, el cual ha perdurado hasta nuestros días, dado que el nombre de leucocitemia propuesto por Bennett (1852) prevaleció sólo por corto tiempo. Virchow diferenció en su trabajo la leucemia de la leucocitosis, describiendo a la vez dos tipos de leucemia: el esplénico, asociado con esplenomegalia, y el linfático, donde se presentaba aumento de tamaño de los ganglios linfáticos. Años después, en 1857, hubo otros aportes novedosos. Friedreich (1857) describió por primera vez una modalidad de leucemia que llamó aguda, y Neumann (1878) estableció la existencia de la leucemia mielógena, conceptos novedosos para aquel tiempo.

Es importante aquilatar el trabajo de estos pioneros en el descubrimiento de la leucemia, sobre todo porque científicamente, la clasificación y el estudio de la leucemia no pudieron visualizarse sino hasta que se conoció la tinción de Erlich (1891) en 1891, la cual permitió diferenciar las distintas etapas madurativas de los leucocitos e identificar las diferentes variantes de las células leucémicas. Resulta también interesante conocer que desde 1903 la leucemia se clasificaba en aguda (linfocítica, mielocítica, monocítica o eritroleucemia) y en crónica (linfocítica o mielocítica) (Reschad y Schilling-Torgau 1913). Sin embargo, no fue sino hasta 1930, al comenzar a realizarse en Europa los aspirados de médula ósea, que empezaron a comprenderse mejor las alteraciones morfológicas de las células madres que producen esta enfermedad (Clemmensen 1974).

\section{ETIOLOGÍA}

Desde las descripciones de Virchow (1845) hasta nuestros días, la etiología de la leucemia ha sido un aspecto controversial entre diversos investigadores, y sus causas han pasado por diferentes etapas: infecciosa, genética, física o química. En el momento actual, después de arduas discusiones entre diversos grupos, debería concluirse que todos los enfoques podrían tener algo de cierto y que la verdadera etiología de la leucemia es probable que se encuentre en la interacción de varias de esas causas.

Causa infecciosa: Desde los primeros casos en el mundo se llegó a definir que las células leucémicas son distintas a las de los procesos inflamatorios o infecciosos. Pese a ello, el interés sobre este punto se ha mantenido hasta el presente, sobre todo porque algunos investigadores han demostrado la presencia de virus como agentes causales de las leucemias humanas y de los animales (Gross 1951).

En 1951, Gross (1951) demostró un virus en una leucemia de los ratones, y posteriormente se aislaron virus en las leucemias de los gatos, monos, ganado vacuno y la leucemia humana T linfocítica (Rickard et al. 1969, Miller et al. 1969, Kawakami et al. 1972, poiesz et al. 1980). Todos esos virus fueron clasificados como retrovirus. Por otra parte, dos virus ADN del grupo del Herpes se han asociado con leucemia: el de la enfermedad de Marek en los pájaros (Churchill y Biggs 1967) y el de Epstein-Barr en la leucemia/linfoma tipo B en los niños africanos (Linfoma de Burkitt) (Epstein et al. 1964).

A pesar de estos informes, la causa infecciosa en otros tipos de leucemia continúa considerándose una hipótesis.

Causa genética: Esta posibilidad fue sugerida por Hartenstein (Engelberth-Holm 1876) en 1896, el cual estudió una vaca y su ternero con leucemia. Durante los años treinta se presentaron informes de leucemia en ratones y su progenie (Slye 1931), lo cual hizo que en años posteriores se encontraran tres familias con miembros que padecían leucemia (Ardashnikov 1937), observándose incluso varios casos en gemelos (MacMahon y Levy 1964). Sin embargo, un estudio en Boston en 1957 no encontró ninguna relación entre parientes (Steinberg 1957), aunque estudios más recientes confirmaron que los hermanos de un niño con leucemia tienen de 2 a 4 veces más probabilidades de desarrollar leucemia que el resto 
de la población infantil (Miller 1971, Draper et al. 1977).

La posibilidad de que el gemelo de un paciente leucémico desarrolle leucemia se encuentra cercana al 25\% (Zuelzer y Cox 1969), habiéndose también identificado un mismo rearreglo genético (inmunoglobulina de cadena pesada) en las células leucémicas de gemelos siameses con leucemia linfocítica aguda (Pombo de Oliveira et al. 1986). En 1993 se identificó el arreglo genético MLL, que involucra la translocación $\mathrm{t}(11 ; 19)(\mathrm{q} 23 ; \mathrm{p} 13)$, en tres pares de gemelos afectados con LLA tipo $\mathrm{T}$, cuyas características fenotípicas incluían $\mathrm{CD}^{+}, \mathrm{CD}^{+}, \mathrm{CD}^{+}$(Ford et al. 1993).

A pesar de que desde 1930 se informó leucemia en el síndrome de Down (Brewster y Cannon 1930), no fue sino hasta 1957 cuando se hizo un estudio amplio sobre esta relación (Krivit y Good 1957). Además, durante los años sesenta comenzó a relacionarse la leucemia infantil con la inmunodeficiencia primaria, con alteraciones cromosómicas y con otros tipos de cáncer (Miller 1967), descubriéndose en esa época el cromosoma Filadelfia en la leucemia mieloide crónica (Rowley 1973).

Otro hallazgo importante dentro de las alteraciones genéticas de las leucemias, fue el descubrimiento del homólogo humano del oncogen $a b l$ del virus de la leucemia murina. Este gen compromete a los cromosomas 9 y 22 , produciendo el gen híbrido BCR-ABL, el cual se encuentra característicamente en la leucemia granulocítica crónica (De Klein et al. 1982).

También es relevante citar que los síndromes de Bloom y de Scwachman, las anemias de Fanconi y de Diamond-Blackfan y la neurofibromatosis, entre otras patologías, presentan un riesgo aumentado de desarrollar leucemia (Baruchel et al. 1999).

Causa física: La historia de María Curie y la leucemia que ella misma desarrolló, siempre se han asociado a la radiación recibida por la investigadora durante sus largos años de estudio. Aun así, no fue sino hasta 1944 cuando esta relación empezó a preocupar a los radiólogos (March 1944). Los estudios en los sobrevivientes de las bombas atómicas en
Japón y las investigaciones subsiguientes en casos de accidentes nucleares, demostraron con claridad que la radiación ionizante en niños puede ser causa inequívoca de leucemia (Simpson et al. 1955).

La perspectiva de que los campos electromagnéticos, generados por las líneas eléctricas de alto voltaje, aumenten el riesgo de leucemias, se presentó como una posibilidad en 1979, pero fue descartada en un trabajo posterior (Campion 1997). Sin embargo este punto continúa en discusión.

Causa química: Aunque a partir de 1928 se había empezado a sospechar de las sustancias químicas como origen de leucemia en adultos con trabajos riesgosos, poco a poco se han incluido otras posibilidades, como la de niños que por viajar en los autos de sus padres con las ventanas cerradas se convierten en fumadores pasivos. Con el advenimiento de la quimioterapia se empezaron a descubrir casos de segundas neoplasias después de los tratamientos anticancerígenos, tanto en niños como en adultos. En la actualidad, los agentes alquilantes, el etopósido (VP-16) y el tenipósido (VM-26) (Tucker et al. 1987) también se incluyen como causas etiológicas de leucemia. Sin embargo, todavía se siguen investigando cuáles sustancias químicas podrían producir leucemia de novo en niños.

Como ya se mencionó, la etiología de las leucemias requiere mayor investigación y no será nada sencillo poder definir muchos casos que presenten esta patología, ya que es probable que un buen número de pacientes tenga etiologías combinadas.

\section{DIAGNÓSTICO DE LAS LEUCEMIAS}

Los análisis de sangre periférica, médula ósea y líquido cefalorraquídeo, con la ayuda de tinciones citoquímicas, son la base del diagnóstico y clasificación de las leucemias (Bennet et al. 1985). Empero, las variaciones morfológicas de las células leucémicas comenzaron a plantear una serie de dificultades diagnósticas en cuanto a su identificación precisa, haciéndose necesario realizar otros estudios 
para clasificar las distintas variantes de ella, entre los cuales pueden citarse los análisis cromosómicos, la citoquímica y el inmunofenotipo, que junto con la edad del paciente, el número de leucocitos y la invasión inicial a otros órganos, permiten definir los factores de riesgo de cada paciente. Otra tecnología que ha ayudado a los pacientes leucémicos han sido los equipos de aféresis, ya que gracias a ellos se consiguió por primera vez transfundir leucocitos, plaquetas o eritrocitos en forma exclusiva.

En la actualidad, la citometría de flujo, la citogenética y los estudios de biología molecular, junto a los análisis tradicionales ya citados, son las principales ayudas para definir el diagnóstico y evaluar el pronóstico y seguimiento de los pacientes con leucemia.

\section{TRATAMIENTO DE LAS LEUCEMIAS}

Como ya se ha mencionado, los primeros tratamientos en esta patología fueron sintomáticos y paliativos. Sin embargo, en la década de los años 40 hubo hallazgos promisorios en este tipo de tratamiento. En primer lugar, el descubrimiento de los antagonistas del ácido fólico por Farber en 1948 permitió obtener remisiones hematológicas que duraron varios meses (Farber et al. 1948). Entre los antagonistas del ácido fólico, el de mayor difusión fue el methotrexate, que rápidamente sustituyó a la aminopterina. La otra droga que revolucionó el manejo de las leucemias fue la hormona adrenocorticotrópica (ACTH) y su análogo la prednisona, con la cual se consiguió un aumento en las remisiones de niños (Farber 1850). La tríada se completó con un antimetabolito de la purina, la 6-mercaptopurina, que ayudó a prolongar las remisiones en los pacientes con leucemia aguda al utilizarse en la llamada fase de mantenimiento (Elion et al. 1951). A partir de ese momento se comenzaron a utilizar diferentes mezclas de drogas, y nuevos medicamentos salieron al mercado con el fin de mejorar los resultados en el tratamiento.

En 1962, el Saint Jude Children's Research Hospital de Memphis, Tennessee, abrió sus puertas con el objetivo de encontrar la cura para los niños leucémicos. Las principales preocupaciones de sus investigadores eran la resistencia a las drogas, generada en algunos pacientes durante la inducción de la remisión inicial; la recaída meníngea, condición asociada a la pobre penetración del medicamento a través de la barrera hematoencefálica; y la toxicidad por el uso de varios medicamentos al mismo tiempo, sobre todo con relación a la hematosupresión, inmunosupresión y mucositis (Pinkel 1999). Con los arduos trabajos de investigación llevados a cabo en ese centro hospitalario, y los abundantes estudios colaborativos que comenzaron a realizarse en el mundo, el panorama cambió radicalmente y muchos pacientes, tanto niños como adultos, empezaron a curarse.

Un gran logro conseguido por las compañías farmacéuticas fue el desarrollo de factores estimulantes de colonias, los cuales han sido importantes en el manejo de los pacientes leucémicos que presentan grandes neutropenias durante su enfermedad.

Como ya se dijo, el diseño del esquema de tratamiento de la leucemia aguda infantil se trasladó a otros tipos de cáncer, logrando con ello la curación de varias formas de neoplasias diseminadas.

\section{LA LEUCEMIA EN COSTA RICA}

Durante las primeras décadas del siglo $\mathrm{XX}$, las principales causas de muerte en nuestro país eran las parasitosis y los problemas infecciosos (Mata 1979). Sin embargo, poco a poco el panorama fue cambiando y en los años 60, al comenzar a disminuirse drásticamente los índices de mortalidad infantil y al controlarse los problemas infecciosos y la desnutrición severa, los accidentes y los tumores malignos empezaron a presentarse como las primeras causas de muerte en el país (Mohs 1980). Por otra parte, debe tenerse presente que la leucemia aguda constituye aproximadamente el 33\% de todas las enfermedades malignas en el niño, lo que les da gran importancia en nuestro medio.

Diagnósticos iniciales: Las leucemias siempre deben haber estado presentes en Costa 
Rica, pero por muchos años su diagnóstico se realizó con temor y casi en forma empírica en el laboratorio clínico del Hospital San Juan de Dios, el primer hospital de caridad fundado en 1845. Ahí, según se dice (C.A. Echandi-Rodríguez 2003, comunicación personal), algunos técnicos de laboratorio al inicio y posteriormente los microbiólogos universitarios, comenzaron a pensar en esa posibilidad diagnóstica al analizar los hemogramas de los pacientes con grandes leucocitosis y con células de morfología anormal. Cabe decir aquí que en la Escuela de Ciencias de la Universidad de Costa Rica, y luego en la naciente Facultad de Microbiología en 1949, se dictaba ya un curso de Hematología.

Primeros hematólogos: En 1960 llegó al país el doctor Jorge Elizondo Cerdas, médico que había realizado en México estudios de especialización en Hematología. Como primer hematólogo nacional, al doctor Elizondo le correspondió hacer los diagnósticos científicos de la leucemia por medio de punciones de médula ósea y por el análisis sistemático de los frotis de sangre periférica.

El doctor Elizondo comenzó así una nueva etapa en Costa Rica, no sólo al introducir los estudios de médula ósea en los pacientes adultos, sino también al aplicar un tratamiento formal para el manejo de estos casos, utilizando los protocolos del Instituto Nacional de la Nutrición de México. Lamentablemente, en esos momentos no se le podía ofrecer a los pacientes ninguna esperanza concreta y la mayoría de ellos morían en pocos meses. El principal interés del hematólogo al diagnosticar una leucemia era manejar adecuadamente las complicaciones infecciosas, sus problemas de sangrado y brindar apoyo al enfermo, buscando también obtener una remisión de su condición hematológica, algo casi imposible para ese tiempo.

Debido a que la labor asistencial del doctor Elizondo se realizaba en el Hospital San Juan de Dios y en el actual Hospital Rafael Ángel Calderón Guardia, él logró formar un Servicio de Hematología en cada uno de esos hospitales de adultos, consiguiendo organizar también un pequeño laboratorio para el estudio de las leucemias y de las otras patologías hematológicas, en el primero de ellos.

En 1966 ocurrió otro hecho en la historia del manejo de las leucemias en nuestro país con la llegada del doctor Elías Jiménez Fonseca, Pediatra Hematólogo formado en el Hospital Infantil de México, quien de inmediato se integró al Hospital Nacional de Niños, recién inaugurado. A partir de ese momento el doctor Jiménez comenzó a hacer estudios de médula ósea en niños, organizando la atención de las leucemias pediátricas.

En 1969 se inauguró el Hospital México y desde entonces su Servicio de Hematología ha manejado las leucemias en ese otro centro.

Laboratorios especializados: Otro episodio en la investigación de la leucemia en Costa Rica es la especialización de los microbiólogos en el campo de la Hematología. El doctor German Sáenz Renauld fue el primero de ellos en interesarse seriamente en la morfología hematológica y desde la Jefatura de su Cátedra de Hematología en la Facultad de Microbiología de la Universidad de Costa Rica, comenzó a enseñar Hematología en forma disciplinada, entusiasmando a sus discípulos.

En el Hospital Nacional de Niños se inauguró en 1974, el Laboratorio de Investigación en Hematología bajo la dirección del doctor Rafael Jiménez Bonilla, quien había estudiado Hematología en el Hospital Infantil de México y en el Saint Thomas's Hospital de Londres, Inglaterra. Desde esa época y hasta la fecha, dicho laboratorio le ha brindado servicio no sólo a los niños de Hematología, sino también a los de Oncología de ese centro hospitalario. Casi al mismo tiempo, el Hospital México creó también un Laboratorio de Hematología. Debe recordarse que el Hospital San Juan de Dios ya tenía el suyo.

Tratamientos modernos: Debido a que en el mundo se estaban curando niños con leucemia (Aur et. al 1971), el doctor Elías Jiménez comenzó a buscar los mejores protocolos de tratamiento para los niños leucémicos, y empezó a indagar la posibilidad de que el Hospital Nacional de Niños se integrara a algún grupo colaborativo de los Estados Unidos. 
En esos mismos años, en febrero de 1973, se realizó en la Habana, Cuba, la Primera Jornada Latinoamericana de Trabajos Cooperativos de Hematología, a la cual asistieron los principales hematólogos latinoamericanos. Durante ese cónclave, el pionero de la Hematología mexicana, el doctor Luis Sánchez Medal, propuso la creación de Grupos Cooperativos de trabajo en las diferentes áreas de la Hematología. Lógicamente, el Grupo Latinoamericano de Tratamiento de las Leucemias y Hemopatías Malignas fue uno de los que se organizó, bajo la dirección del médico argentino doctor Santiago Pavlovsky, y los hospitales de Costa Rica (el México, el San Juan de Dios y el Nacional de Niños) se adhirieron a sus protocolos a partir de 1979. De esta forma se comenzaron a introducir en Costa Rica los tratamientos con múltiples drogas, con lo cual los pacientes leucémicos consiguieron prolongar sus remisiones clínicas.

No puede dejar de considerarse la importancia que para la atención de los niños leucémicos tuvieron las asesorías dadas por los médicos del Saint Jude Children's Research Hospital al Hospital Nacional de Niños, lo que repercutió no sólo en el uso de modernos protocolos de tratamiento, sino también en la conformación de un grupo multidisciplinario de profesionales para la atención y cuidado de los niños con cáncer.

En la actualidad se cuenta con diferentes ayudas internacionales que contribuyen a mejorar en nuestros hospitales el tratamiento y el diagnóstico de los pacientes con leucemia.

Otro de los pasos novedosos en el manejo de las leucemias en Costa Rica fue el inicio de los transplantes de médula ósea, el primer país centroamericano que emprendió este arriesgado procedimiento. Los transplantes se iniciaron en 1985 en el Hospital Nacional de Niños, gracias a la ayuda del Servicio de Inmunología del Hospital de Gotemburgo, Suecia, que junto con el personal de los Servicios de Inmunología y Hematología del Hospital Nacional de Niños, fueron los artífices en implantar esta novedosa opción, no sólo para los pacientes con neoplasias hematológicas sino para otros tipos de patologías infantiles, como las inmunodeficiencias primarias, la osteopetrosis y la anemia aplástica. El quinto transplante de médula ósea en Costa Rica fue a una niña con leucemia linfocítica aguda, la cual recibió en 1988 su médula de una gemela idéntica. Posteriormente, en mayo de 1992, el Hospital San Juan de Dios empezó su programa de transplantes de médula ósea en los adultos, teniendo en este momento la cifra de veintiún transplantes en leucemias. También el Hospital México inició en 1995 su programa de transplantes medulares, realizando en 1998 el primer transplante autólogo en el país, y en el 2002 el primero con células de cordón umbilical. En el momento actual, dicho hospital tiene la cifra récord de 56 transplantes medulares realizados. Estos procedimientos han ayudado a prolongar la vida de muchos pacientes y en algunos casos han conseguido la remisión permanente de los leucémicos.

Relaciones internacionales: La unión de los Servicios de Hematología nacionales con el Grupo Latinoamericano de Tratamiento de las Leucemias y Hemopatías Malignas, y la relación del Saint Jude Children's Research Hospital y del National Cancer Institute con el Hospital Nacional de Niños, trajeron al país grandes beneficios. Por una parte, se fortalecieron los tratamientos multidrogas, los cuales consiguieron prolongar las remisiones clínicas y obtener las primeras curaciones de leucemia en el país, pero a la vez estas relaciones produjeron otros logros relevantes. Debido a que en los Estados Unidos, para realizar protocolos de tratamiento con diferentes drogas en niños leucémicos se obligaba a los hospitales de aquel país a tener Comités de Bioética, el Hospital Nacional de Niños debió conformar un Comité de Bioética e Investigación, el primero en el país, el cual fue organizado con los mayores rigores del momento: personas de alta categoría moral, miembros de áreas médicas y no médicas, representantes de la comunidad e igual número de hombres y mujeres. De esa forma, el manejo de los pacientes con leucemia logró que Costa Rica se pusiera a la altura de los países desarrollados que comenzaban a preocuparse 
por proteger los derechos de los niños. Otro aspecto interesante es que por un error, el National Cancer Institute designó como hospital hermano del Hospital Nacional de Niños al Baylor Medical Center, el cual resultó ser un centro de adultos. Por este motivo, los hematólogos del Hospital Nacional de Niños le pidieron al Hospital San Juan de Dios que se hermanara con Baylor. Posteriormente, gracias a la colaboración de ese centro norteamericano, el Hospital Nacional de Niños se hermanó con el Dallas Pediatric Hospital, lo que significó a la postre muchos beneficios para Costa Rica.

Como ya se dijo, una de las relaciones más fructíferas para el manejo de los pacientes leucémicos en edad pediátrica fue la asesoría brindada al Hospital Nacional de Niños por diversos especialistas del Saint Jude Children's Research Hospital. Dicho centro había comenzado a utilizar en los niños norteamericanos un tratamiento total, que incluía diversas drogas para la inducción de la remisión, seguida de cursos de quimio y radioterapia para la prevención de recaída al sistema nervioso central. Las visitas de los hematólogos del Saint Jude a Costa Rica hicieron posible la creación de un centro nacional multidisciplinario para el manejo de los niños con leucemia, el cual contó con visitas de entrenamiento para médicos, microbiólogos y enfermeras costarricenses, y sobre todo, mantuvo una discusión permanente para el uso de los protocolos de tratamiento más adecuados para cada tipo de leucemia. Esas visitas, más la preocupación de algunos padres costarricenses de niños enfermos, sirvieron para organizar un grupo de personas que ayudara económicamente a los Servicios de Hematología y Oncología, así como al Laboratorio de Investigación del Hospital Nacional de Niños. Estos voluntarios se dedicaron a brindar también apoyo emocional a los pacientes y a sus familiares, y a conseguir dinero para estos fines. De esa forma nació la Asociación Lucha Contra el Cáncer Infantil, ejemplo de Costa Rica hacia el mundo. A partir de su fundación en 1980, la deserción al tratamiento de los niños leucémicos del Hospital Nacional de Niños disminuyó a cifras inferiores al 3\%, y poco a poco se comenzó a curar un número mayor de niños, gracias a los modernos protocolos aplicados y al apoyo de esa Asociación, la cual empezó a pagar los pasajes de buses a los niños y padres provenientes de zonas rurales, así como a sufragar parte de los gastos del encargado en llevar al niño a sus consultas médicas. En 1993, la Asociación Lucha Contra el Cáncer Infantil logró construir un albergue cercano al Hospital Nacional de Niños, donde hasta la fecha se hospedan y alimentan -sin costo alguno- los pequeños pacientes y su acompañante.

En 1996, la Sección de Hematología Pediátrica del Hospital San Gerardo el Nuevo de Monza, Italia, se propuso mejorar el manejo integral y el tratamiento de los tumores infantiles en Centro América, para lo cual becó a distintos profesionales médicos y de laboratorio para que se entrenaran en sus instalaciones. Producto de esta ayuda se fundó en 1999 la Asociación de Hemato-Oncología Pediátrica Centroamericana, la cual ha organizado desde su creación reuniones anuales en los distintos países de Centro América, con el fin de intercambiar conocimientos y de discutir los protocolos de tratamiento de las diferentes patologías malignas en niños.

Implementación de nuevas metodologías: Al mismo tiempo que los laboratorios de Hematología comenzaron a atender los casos de pacientes con leucemia y a clasificarlos por medio de la morfología celular y de la citoquímica avanzada, la metodología para su diagnóstico empezó a sofisticarse, haciéndose necesario que el país contara con una citocentrífuga. La primera la adquirió el Laboratorio de Investigación del Hospital Nacional de Niños. También en ese mismo laboratorio se implementaron tinciones especiales para identificar parásitos como el Pneumocystis carinii. Posteriormente, en el Hospital Nacional de Niños se comenzaron a realizar estudios cromosómicos en los niños con leucemia, y a principios de la década de los noventa su Laboratorio de Investigación envió a capacitar al extranjero a una microbióloga para que se dedicara a hacer estudios de citometría de flujo, 
no sólo para la clasificación inmunofenotípica de las leucemias sino para el seguimiento de los pacientes con el nuevo concepto de recaída inmunológica, analizada mediante los estudios de Enfermedad Mínima Residual.

Cursos de Especialización: A final de los años setenta, la Asociación Costarricense de Hematología comenzó a dictar cursos de actualización en leucemias, para lo cual trajo al país a destacados especialistas internacionales, los cuales vinieron a brindar sus conocimientos.

Por otra parte, el Hospital Nacional de Niños inició en 1975 la especialización de médicos en Hematología Pediátrica, y la Universidad de Costa Rica comenzó en 1977 un fructífero programa de Especialización en Hematología para microbiólogos. También el Hospital San Juan de Dios empezó a especializar médicos en Hematología de adultos. En la actualidad, los jóvenes profesionales graduados de esos cursos son los que manejan los centros de atención de la leucemia en el país.

Trabajos de investigación: La introducción de novedosos métodos de tratamiento hizo que se hiciera necesario evaluar los resultados obtenidos en los adultos y niños. Por lo tanto, los diferentes hospitales comenzaron a presentar protocolos de estudio y manejo para los pacientes leucémicos. Resulta muy satisfactorio para la Hematología, que el primer proyecto financiado por el Consejo Nacional de Investigación en Ciencia y Tecnología, fuera un protocolo para la atención de niños con leucemia presentado por el Servicio de Hematología del Hospital Nacional de Niños.

En el año 2001, la Florida Ice \& Farm promovió un concurso de proyectos de investigación con el fin de donar fondos para promover esta iniciativa en el país. El proyecto de estudios de biología molecular en las leucemias infantiles, presentado por los investigadores del Centro de Investigación en Hemoglobinas Anormales y Trastornos Afines de la Universidad de Costa Rica, en conjunto con el Servicio de Hematología del Hospital Nacional de Niños, ganó el primer premio en ese concurso. Este hecho representó la primera vez que una empresa nacional privada ha financiado proyectos de investigación, ejemplo que debería ser emulado por otras empresas locales.

Publicaciones costarricenses: Los investigadores costarricenses, clínicos y de laboratorio, han publicado sus experiencias en el tratamiento y diagnóstico de las leucemias. En este apartado no se pretende hacer una revisión completa de todas las publicaciones nacionales en este campo y sólo se anotarán algunas de las realizadas a lo largo de más de treinta años de trabajo.

En 1970, Jiménez et al. registraron una incidencia de la leucemia infantil de 2.5 casos por cada 100000 habitantes, valor que subió a 4.1 por cada 100000 habitantes al final de la década de los setenta (Anónimo 1980).

Algunos ejemplos de los estudios cromosómicos en leucemias son las publicaciones de Castro Volio et al. (1993) y de Solís et al. (2000), realizadas la primera en el Hospital México y en el INISA, y la segunda en el Nacional de Niños y Calderón Guardia, así como las hechas por Venegas y Rivera (2001a, b, 2004) en el Hospital Nacional de Niños, en las que se han informado la presencia del cromosoma Philadelphia en un alto porcentaje de las leucemias granulocíticas crónicas, así como el hallazgo de la traslocación $\mathrm{t}(4 ; 11)$ en niños con leucemia linfocítica aguda (Venegas 2001b), factor asociado a pobre pronóstico (ver además Castro Volio 2004).

En adultos se han publicado las experiencias tanto en las leucemias agudas como en las crónicas (Montero et al. 1974, Elizondo et al. 1987, Vásquez et al. 1988), y en niños se ha producido gran cantidad de artículos donde se han analizado la importancia de la citocentrífuga (Jiménez et al. 1977) y de los cambios proteicos y clínicos en las recaídas meníngeas (Jiménez et al. 1978, 1981), los resultados del tratamiento en los pacientes pediátricos (Jiménez et al. 1979) y con alto riego (Jiménez et al. 1975), los hallazgos en las complicaciones infecciosas (Jiménez et al. 1975, Carrillo et al. 1981) y los cambios de la coagulación sanguínea en ese tipo de pacientes (Jiménez et al. 1981).

Un artículo con gran difusión internacional, que analizó tanto niños como adultos, fue 
el publicado por Jiménez en 1981, en el cual se presentaron los datos de leucemia y linfomas en la América Tropical.

Futuro de las leucemias en Costa Rica: Aunque es muy difícil predecir qué pasará en los próximos años con el diagnóstico y manejo de esta patología en nuestro país, es muy probable que la Caja Costarricense de Seguro Social llegue a introducir a corto plazo las técnicas de biología molecular y de hibridización in situ, con las cuales se individualizarán los tipos de leucemia y los riesgos de recaída para cada paciente. También es posible que lleguen a utilizarse fármacos complejos que incluyan anticuerpos específicos o que hayan sido producidos mediante tecnología recombinante. También el mejor uso de los transplantes de médula ósea, con métodos novedosos, hará más fácil el tratamiento de algunos casos con este problema.

Todo esto, aunado a los modernos métodos diagnósticos que de seguro aparecerán en los años venideros, hará que se planifique un tratamiento personalizado para cada paciente, tomándose en cuenta los factores pronósticos y el tipo de leucemia que presente.

\section{RESUMEN}

Se hace inicialmente una revisión de la historia de la leucemia en el mundo, analizando sus principales causas etiológicas y las primeras formas de diagnóstico y tratamiento. A continuación se revisa la historia de la investigación de la leucemia en Costa Rica, puntualizando los diagnósticos iniciales en los hospitales, la llegada de los primeros hematólogos clínicos y de laboratorio, la creación de los laboratorios especializados en Hematología, la aplicación en nuestro país de los modernos tratamientos contra la leucemia y la importancia que para ello tuvieron las relaciones con centros del extranjero. También se presenta la secuencia de la implementación en Costa Rica de las modernas metodologías de ayuda diagnóstica y la importancia que tuvo en el manejo de la leucemia el hecho de que se empezaran a formar en el país nuevos profesionales especializados en la rama de la Hematología, así como la creación de las asociaciones de voluntarios. Por último se presentan algunas de las publicaciones nacionales en leucemia y unos comentarios acerca del futuro del tratamiento y el diagnóstico de los pacientes leucémicos.

\section{REFERENCIAS}

Anónimo. 1980. Tumores malignos del tejido linfático y de los órganos hematopoyéticos. Departamento de Estadística, Ministerio de Salud, Costa Rica.

Ardashnikov, S.N. 1937. The genetics of leukemia in man. J. Hyg. 37:286-302.

Aur, R.A.J., J.V. Simone, H.O. Hustu, T. Walters, L. Borella, C. Pratt \& D. Pinkel. 1971. Central nervous system therapy and combination chemotherapy of childhood lymphocytic leukemia. Blood 37: $272-281$.

Barth, C. 1856. Altération du sang remarquable par la prédominance des globules blancs ou musqueux ; hypertrophie considérable de la rate. Bull. Soc. Méd. Hôp., Paris. 39 p.

Baruchel, A., T. Leblanc \& G. Schaison. 1999. Pathology of acute lymphoblastic leukemia. In J. Lilleyman, I. Hann \& V. Blanchette (eds.). Pediatric Hematology. Churchill Livingston, London, England. 520 p.

Bennett, J.H. 1845. Case of hypertrophy of spleen and liver in which death took place from suppuration of blood. Edinburgh Med. Surg. J. 64: 413-423.

Bennett, J.H. 1852. Leucocythaemia or white cell blood. Sutherland and Knox, Edinburgh.

Bennet, J.M., D. Catowsky, M.T. Daniel, G. Flandrin, D.A.G. Galton, H.R. Gralnick \& C. Sultan. 1985. Proposed revised criteria for the classification of acute Mieloid Leukemia. A report of the FrenchAmerican-British co-operative group. Ann. Int. Med. 100: 626-629.

Brewster, H.F. \& H.E. Cannon. 1930. Acute lymphatic leukemia: report of a case in an eleventh month mongolian idiot. New Orleans Med. Surg. J. 82: 872-873.

Campion, E.W. 1997. Power lines, cancer and fear. N. Engl. J. Med. 337: 44-46.

Carrillo, J.M., E. Jiménez, M. Navarrete, R. Jiménez, I. García, J.F. Lobo \& L.A. Mora. 1981. Enfermedades infecciosas en niños con cáncer. Sangre 26: 15-26.

Castro Volio, I., C. Montero Umaña \& G. Jiménez Cruz. 1993. Características cromosómicas asociadas con leucemias y otras hemopatías en Costa Rica. Rev. Biol. Trop. 41: 385-392.

Castro Volio, I. 2004. Pasado, presente y futuro de la citogenética humana en Costa Rica. Rev. Biol. Trop. 52(3): 133-140. 
Churchill, A.E. \& P.M. Biggs. 1967. Agent of Marek's disease in tissue culture. Nature 215: 528-530.

Clemmensen, J. 1974. On the epidemiology of leukemia, pp. 1-50. In F.J. Cleton, D. Crowther \& J.S. Malpas (eds.). Advances in acute leukemia. North Holland, Elsevier.

Craigie, D. 1845. Case of disease of spleen in which death took place in consequence of the presence of purulent matter in the blood. Edinburgh Med. Surg. J. 400-413.

Dameshek, W. \& F. Gunz. 1964. Leukemia. 1 p. Grune \& Stratton, New York/London.

De Klein, A., A.G. Van Kessel, G. Grosveld, C.R. Bartram, A. Hagemeijer, D. Bootsma, N.K. Spurr, N. Heisterkamp, J. Groffen \& J.R. Stephenson. 1982. A cellular oncogene is translocated to the Philadelphia chromosome in chronic myelocytic leukemia. Nature. 300 : 765-767.

Donné, A. 1844. Cours de microscopie. J.B. Baillière, Paris. $132 \mathrm{p}$.

Draper, G.J., M.M. Heaf \& L.M. Kennier-Wilson. 1977. Occurrence of childhood cancers among sibs and estimation of familial risk. J. Med. Genet. 14: 81-90.

Ehrlich, P. 1891. In A. Hirschwald (ed.). Farbenanalytische Untersuchungen zur Histologie und Klinick des Blutes. Berlin, A. Hirschwald. 137 p.

Elion, G.B., G.H. Hitchings \& H. Vanderwerff. 1951. Antagonists of nucleic acid derivatives. VI. Purines. J. Biol. Chem. 192: 505-518.

Elizondo Cerdas, J., L. Salazar Sánchez, M. Ramón Ortiz \& L.F. Vásquez Castillo. 1987. Leucemia linfocítica crónica. Experiencia acumulada en el Servicio de Hematología del Hospital San Juan de Dios. Rev. Cost. Cienc. Méd. 8(4): 281-284.

Engelberth-Holm, J. 1876. Hartenstein, Ver Veteriärw, Sachsen. In Oliver and Boyd (eds.). Spontaneous and experimental leukemia in animals. Edinburgh. 44 p.

Epstein, M.A., B.G. Achong \& Y.M. Barr. 1964. Virus particles in cultured lymphoblasts from Burkitt's lymphoma. Lancet. 1: 702-703.

Farber, S., L.K. Diamond, R.D. Mercer, et al. 1948. Temporary remissions in acute leukemia in children produced by folic acid antagonist, 4-aminopteroylglutamic acid (aminopterin). N. Engl. J. Med. 238: 787-793.

Farber, S. 1850. The effect of ACTH in acute leukemia in children. In J.R. Mote (ed.). First clinical ACTH conference. New York, Blakiston. 325 p.
Ford, A.M., S.A. Ridge, M.E. Cabrera, H. Mahmoud, C.M. Steel, L.C. Chan \& M. Greaves. 1993. In utero rearrangements in the trithorax-related oncogene in infant leukaemias. Nature 363: 358-360.

Friedreich, N. 1857. Ein neuer Fall von Leukäemie. Virchow's Arch. Pathol. Anat. 12: 37-58.

Gross, L. 1951. "Spontaneous" leukemia developing in $\mathrm{C}_{3} \mathrm{H}$ mice following inoculation, in infancy, with $\mathrm{AK}$ leukemic extracts or AK embryos. Proc. Soc. Exp. Biol. Med. 76: 27-32.

Jiménez, E. 1981. Lymphomas and leukemias in Tropical America. Clin. Haemat. 10: 894-915.

Jiménez, R.A., A. Apéstequi, E. Jiménez, R. Trejos, L.A.Mora, J.M. Carrillo, M. Navarrete \& J.F. Lobo. 1981. Protein changes in cerebrospinal fluid of children with meningeal leukemia. Acta Hematol. 65: 48-53.

Jiménez, E., J.M. Carrillo, R. Jiménez, J.F. Lobo \& A. Camacho. 1979. Tratamiento de niños con leucemia linfocítica aguda en Costa Rica. Bol. of Sanit. Panam. 87: 525-534.

Jiménez, E., R. Jiménez, E. Quesada, F. Lobo \& M. Granados. 1975. Tratamiento del niño con leucemia linfocítica aguda considerado como de alto riesgo. Rev. Méd. Hosp. Nac. de Niños 10: 113-118.

Jiménez, R., E. Jiménez, L.A. Mora, M. Navarrete, J.M. Carrillo \& F. Lobo. 1981. Alteraciones de la hemostasia en niños con problemas neoplásicos. Sangre 26: $38-46$.

Jiménez, E., M. Martínez, E. Quesada, J. Elizondo, M. Zomer \& R. Cordero. 1970. Estudio cooperativo de leucemias en Costa Rica. Acta Méd. Cost. 13: 43-52.

Jiménez, R., L.A. Mora, E. Jiménez, J.M. Carrillo \& F. Lobo. 1977. Estudio de células tumorales en los líquidos cefalorraquídeos mediante citocentrifugación. Sangre 22: 953-960.

Jiménez, R., L.M. Mora, E. Jiménez, M. Navarrete \& J.F. Lobo. 1978. Significado de la pleocitosis del LCR en niños con neoplasias. Bol. Méd. Hosp. Inf. (Méx) 36: $331-336$

Jiménez, E., E. Quesada, F. Lobo \& R. Jiménez. 1975. Características de las enfermedades exantemáticas en niños con cáncer y en tratamiento con inmunosupresores. Rev. Méd. Hosp. Nac. de Niños 10: 119-126.

Kawakami, T.G., S.D. Huff \& P.M. Buckley, D.L. Dungworth, S.P. Synder, R.V. Gilden. 1972. C-type virus associated with gibbon lymphosarcoma. Nature (New Biol) 235: 170-171. 
Krivit, W. \& Good Ra. 1957. Simultaneous occurrence of mongolism and leukemia. J. Dis. Child (AMA) 94: 289-293.

MacMahon, B. \& M.A. Levy. 1964. Prenatal origin of childhood leukemia. Evidence from twins. N. Engl. J. Med. 270: 1082-1085.

March, H.C. 1944. Leukemia in radiologists. Radiology 43: 275-278.

Mata, L. 1979. The malnutrition-infection complex and its environment factors. Proc. of the Nutr. Soc. 38: 29-40.

Miller, R.W. 1967. Persons with an exceptionally high risk of leukemia. Cancer Res. 27: 2420-2423.

Miller, R.W. 1971. Deaths from childhood leukemia and solid tumors among twins and other sibs in the United States. J. Natl. Cancer Inst. 46: 203-209.

Miller, J.M., L.D. Miller, C. Olson \& K.G. Gillette. 1969. Virus-like particles in phytohemagglutinin-stimulated lymphocyte cultures with reference to bovine lymphosarcoma. J. Natl. Cancer Inst. 43: 1297-1305.

Mohs, E. 1980. La Pediatría Nacional. Etapas y logros. In Salud, Medicina y Democracia. Universidad de Costa Rica. 171 p.

Montero, C., A. Barrantes \& R. Cordero. 1974. Evaluación clínica de la quimioterapia en la leucemia mieloblástica. Rev. Méd. (Méx). 54: 508-513.

Neumann, E. 1878. Ueber myelogene Leukäemie. Ber Klin Wochenschr 15: 69-72.

Pinkel, D. 1999. Historical perspective. In C.H. Pui (ed.). Childhood leukemias. Cambridge University, USA. 9 p.

Poiesz, B.J., F.W. Ruscette \& A.F. Gagdar, P.A. Bunn, J.D. Minna \& R.C. Gallo. 1980. Detection and isolation of type $\mathrm{C}$ retrovirus particles from fresh and cultured lymphocytes of a patient with cutaneous T-cell lymphoma. Proc. Natl. Acad. Sci. USA. 77: 7415-7419.

Pombo de Oliveira, M.S., F.E. Awad el Seed, L. Foroni, E. Matutes, R. Morilla, L. Luzzatto \& D. Catovsky. 1986. Lymphoblastic leukemia in Siamese twins: evidence for identity. Lancet. ii: 969-970.

Reschad, H. \& V. Schilling-Torgau. 1913. Ueber eine neue Leukäemie durch echte Uebergangsformen und ihre bedeutung für die Selbständigkeit dieser zellen. Munch Med Wochenschr. 60: 1981-1984.

Rickard, C.G., J.E. Post, F. Noronha \& L.M. Barr. 1969. A transmissable virus-induced lymphocytic leukemia of the cat. J. Natl. Cancer Inst. 42: 987-1014.

Rowley, J.D. 1973. A new consistent chromosome abnormality in chronic myelogenous leukemia identified by quinacrine fluorescence and Giemsa staining. Nature 243: 290-293.

Simpson, C.L., L.H. Hempelman \& L.M. Fuller. 1955. Neoplasia in children treated with X-rays in infancy for thymic enlargement. Radiology 64: 840-845.

Slye, M. 1931. The relation of heredity to the occurrence of spontaneous leukemia, pseudoleukemia, lymphosarcoma and allied diseases in mice. Preliminary report. Am. J. Cancer 15: 1361-1386.

Solís, M.V., M.A. Alvarado, E. Ruiz, J.M. Carrillo, M. Navarrete, G. Sánchez \& E. Jiménez. 2000. Citogenética e histoquímica de pacientes con leucemia en dos hospitales neotropicales. Rev. Biol. Trop. 48: 707-718.

Steinberg, A.G. 1957. A genetic and statistical study of acute leukemia in children, pp. 353-356. In Proceedings of the third national cancer conference. J.B. Lippincott, Philadelphia.

Tucker, M.A., A.T. Meadows, J.D. Boice Jr, M. Stovall, O. Oberlin, B.J. Stone, J. Birch, P.A. Voute, R.N. Hoover, \& J.F. Fraumeni Jr. 1987. Leukemia after therapy with alkylating agents for childhood cancer. J. Natl. Cancer Inst. 78: 459-464.

Vásquez, L.F., M. Ramón \& L. Salazar. 1988. Leucemia aguda no linfocítica con expresión de marcadores citoquímicos de serie granulocítica y monocítica en la misma célula. Reporte de un caso. Rev. Cost. Cienc. Méd. 9: 108-111.

Velpeau, A. 1827. Sur la resorption du pusaet sur l'alteration du sang dans les maladies clinique de persection nenemant. Premier observation. Rév. Méd. 2: 216.

Venegas, P. 2001a. Interfase-FISH en un paciente con leukemia mielocítica crónica. Rev. Méd. Hosp. Nac. de Niños 36: 9-13.

Venegas, P. 2001b. Translocación $\mathrm{t}(4 ; 11)$ de infantes con leucemia linfocítica aguda. Rev. Méd. Hosp. Nac. Niños 36: 5-8.

Venegas, P. \& J. Rivera. 2004. Estudios citogeneticos en niños con leucemia linfocitica aguda-B en Costa Rica. Rev. Biol. Trop. 52(3): 147-154.

Virchow, R. 1845. Weisses Blut. Notiz Geb Natur Heilk. 36: $152-156$.

Virchow, R. 1856. Die Leukäemie, pp. 190-211. In R. Virchow (ed.). Gesammelte Abhandlungen zur Wiessenschaft lichen Medizin. Frankfurt, Meideinger.

Zuelzer, W. \& D. Cox. 1969. Genetic aspects of leukemia. Semin. Hematol. 6: 228-249. 